\title{
Numerical Well Test Analysis for Polymer Flooding considering the Non-Newtonian Behavior
}

\author{
Jia Zhichun, ${ }^{1}$ Li Daolun, ${ }^{1,2}$ Yang Jinghai, ${ }^{3}$ Xue Zhenggang, ${ }^{1}$ and Lu Detang ${ }^{1}$ \\ ${ }^{1}$ University of Science and Technology of China, Hefei, Anhui 230026, China \\ ${ }^{2}$ Hefei University of Technology, Hefei, Anhui 230026, China \\ ${ }^{3}$ Daqing Oilfield Company Ltd., Daqing, Heilongjiang 163453, China \\ Correspondence should be addressed to Li Daolun; ldaol@ustc.edu.cn
}

Received 10 October 2014; Revised 6 January 2015; Accepted 11 February 2015

Academic Editor: Sachin Jangam

Copyright (C) 2015 Jia Zhichun et al. This is an open access article distributed under the Creative Commons Attribution License, which permits unrestricted use, distribution, and reproduction in any medium, provided the original work is properly cited.

Well test analysis for polymer flooding is different from traditional well test analysis because of the non-Newtonian properties of underground flow and other mechanisms involved in polymer flooding. Few of the present works have proposed a numerical approach of pressure transient analysis which fully considers the non-Newtonian effect of real polymer solution and interprets the polymer rheology from details of pressure transient response. In this study, a two-phase four-component fully implicit numerical model incorporating shear thinning effect for polymer flooding based on PEBI (Perpendicular Bisection) grid is developed to study transient pressure responses in polymer flooding reservoirs. Parametric studies are conducted to quantify the effect of shear thinning and polymer concentration on the pressure transient response. Results show that shear thinning effect leads to obvious and characteristic nonsmoothness on pressure derivative curves, and the oscillation amplitude of the shear-thinning-induced nonsmoothness is related to the viscosity change decided by shear thinning effect and polymer concentration. Practical applications are carried out with shut-in data obtained in Daqing oil field, which validates our findings. The proposed method and the findings in this paper show significant importance for well test analysis for polymer flooding and the determination of the polymer in situ rheology.

\section{Introduction}

Polymer flooding is one of the most mature EOR (Enhanced Oil Recovery) techniques used in oilfields [1]. It is providing more than $30 \%$ of the total oil production in China's Daqing oilfield today. In polymer flooding, polymer solutions injected into the reservoir exhibit non-Newtonian properties $[2,3]$. A number of studies have discussed the influence of non-Newtonian behavior of polymer on sweep efficiency and recovery, and the results proved the importance of taking polymer rheology into account for the successful design and evaluation of polymer flooding project [4-6].

The estimation of in situ rheology of polymer solutions in porous medium is difficult in practice [7]. Early time researches in chemical engineering, rheology, and petroleum engineering have focused on the flow behavior of nonNewtonian fluids in porous medium [8-10]. However, the in situ polymer rheology may not consist with the results of those experimental and analytical researches due to the great differences between the actual situation and the simplified and idealized conditions. One approach to directly determine the polymer in situ rheology is by way of well test analysis. The results of several previous works indicated that the well test data from polymer flooding wells can be interpreted to determine the in situ viscosity of non-Newtonian polymer solution [7].

Well test analysis, which is widely used in oilfields, provides valuable information of formation and fluid underground by interpreting the pressure transient response [11]. Yet, conventional well test interpretational models do not work for reservoirs containing non-Newtonian fluids, and well test analysis for polymer flooding is more difficult because of the treatment of polymer rheology and other complicated mechanisms involved in polymer flooding process [11-13]. 
Researchers have exerted their efforts on well test analysis for non-Newtonian fluid since the 1970s. Ikoku and Ramey [14] and Odeh and Yang [15] proposed mathematical models for flow of non-Newtonian power-law fluid in homogeneous porous medium and induced methods of well test analysis for non-Newtonian fluids. Vongvuthipornchai and Raghavan [16] developed type curves which includes wellbore storage and skin factor for well test analysis for non-Newtonian fluid. Katime-Meindl and Tiab [13] developed the TDS (Tiab's direct synthesis) method for well test analysis and used it for interpretation of non-Newtonian fluid. Recently, Escobar and Martinez et al. [17, 18] applied the TDS technique to pseudoplastic and dilatants fluids in a radial composite reservoir. Yu et al. [19] established a well testing model for polymer flooding based on rheology experiments and presented a numerical well testing interpretation model and analysis techniques to evaluate formation by using pressure transient data in cross-flow double-layer reservoirs. Another recent research of the well test analysis for polymer flooding was performed by $\mathrm{Yu}$ et al. [19], presenting a numericalanalytical combined method to infer the in situ polymer rheology from PFO (Pressure Fall-Off) tests.

These works were remarkable and they promoted our understanding of the pressure transient response in polymer flooding. However, despite decades of research, the existing methods of well test analysis for polymer flooding are far from satisfactory for field application. Reasons are as follows: firstly, most of the previous works are analytical approaches, which is mostly confirmed to dealing with certain simplified situations and have limitations in complex field applications. Secondly, the results reported in those papers do not fully describe certain behaviors of actual measured data from polymer flooding wells. Finally, few field examples have been presented which are studied and interpreted to validate the analytical or numerical results.

In this study, a two-phase four-component polymer flooding model is given considering the major physicochemical phenomena including polymer thickening, shear thinning, polymer adsorption, permeability reduction, and dead pore volume. A fully implicit iterative numerical simulator is developed based on PEBI gridding to study the transient pressure response for wells in polymer flooding reservoirs. Parametric studies were conducted to quantify the effect of shear thinning and polymer concentration on the pressure transient response. Finally, a field example was studied in which two pressure build-up tests were performed on the same oil producer from a polymer flooding reservoir in Daqing oilfield. Reservoir parameters and the polymer in situ shear thinning properties were interpreted for the real example, and the impact of adjusting shear thinning on pressure derivative curves is discussed.

\section{Methodology}

2.1. Basic Equations for Polymer Flooding. To build the mathematical model for polymer flooding, several assumptions were made to simplify the real situation. The major assumptions in this paper are as follows: (1) the reservoir is isothermal; (2) Darcy's law for fluid flow in porous medium applies; (3) the fluid underground contains two phases: the water phase and the oil phase. The water phase contains three components: pure water, polymer, and salt. (4) The process of polymer adsorption is irreversible. (5) No chemical reaction occurs. (6) Diffusion and dispersion are not considered.

Based on the assumptions above, the mass balance equations were given for each component. Considering the physicochemical phenomena including polymer thickening, polymer adsorption, permeability reduction, and dead pore volume, the basic equations of the polymer flooding model are given by

$$
\begin{gathered}
\text { Oil: } \nabla \cdot\left[\frac{K K_{r o}}{\mu_{o} B_{o}}\left(\nabla p_{o}-\gamma_{o} \nabla D\right)\right]+q_{o}=\frac{\partial}{\partial t}\left(\frac{\phi S_{o}}{B_{o}}\right) \\
\text { water: } \nabla \cdot\left[\frac{K K_{r w}}{R_{k} \mu_{w} B_{w}}\left(\nabla p_{w}-\gamma_{w} \nabla D\right)\right]+q_{w}=\frac{\partial}{\partial t}\left(\frac{\phi S_{w}}{B_{w}}\right) \\
\text { polymer: } \nabla \cdot\left[\frac{K K_{r w}}{R_{k} \mu_{w} B_{w}}\left(\nabla p_{w}-\gamma_{w} \nabla D\right) C_{p}\right] \\
+q_{p}=\frac{\partial}{\partial t}\left[\frac{\phi_{p}\left(S_{w} C_{p}+C_{p a d s}\right)}{B_{w}}\right] \\
\text { salt: } \nabla \cdot\left[\frac{K K_{r w}}{R_{k} \mu_{w} B_{w}}\left(\nabla p_{w}-\gamma_{w} \nabla D\right) C_{\mathrm{sa}}\right] \\
+q_{c l}=\frac{\partial}{\partial t}\left(\frac{\phi S_{w} C_{\mathrm{sa}}}{B_{w}}\right) .
\end{gathered}
$$

To enclose the equations, additional relations were induced including the saturation relation and the capillary pressure relation.

Saturation relation:

$$
S_{o}+S_{w}=1
$$

Capillary pressure relation:

$$
p_{c o w}=p_{o}-p_{w}=p_{c}\left(S_{w}, \sigma_{w o}\right) .
$$

The Peaceman model was used to deal with the wells. Let $Q$ be the production of the well at surface condition, considering the wellbore storage, the flow equation in the wellbore is given by

$$
\frac{1}{\mu_{l} B_{l}} \frac{2 \pi K_{r l} K h}{\ln \left(r_{\text {eff }} / r_{w}\right)+S}\left(p-p_{w f}\right)-\frac{C_{\text {wellbore }}}{\Delta t}\left(p_{w f}^{n+1}-p_{w f}^{n}\right)=Q \text {. }
$$

Equations (1)-(4) are discretized based on PEBI grid by control volume method. And all the unknowns are solved fully implicitly by GMRES matrix solver in this study.

2.2. Modeling the Water Phase Viscosity. In the polymerflooding model, the water phase contains three components: pure water, polymer, and salt. The water phase viscosity is a function of polymer concentration, salinity, and shear rate [2]. The water phase viscosity is calculated following 
the steps below. Firstly, the unsheared water phase viscosity $\mu_{0}$ is determined according to the polymer concentration and salinity using

$$
\mu_{0}=\mu_{w} \cdot \mu_{p}
$$

where $\mu_{w}$ denotes the viscosity of pure water and $\mu_{p}$ is a viscosity multiplier which is a function of polymer concentration and salinity;

$$
\mu_{p}=\mu_{p}\left(C_{p}, C_{\mathrm{sa}}\right),
$$

where $C_{p}$ is the polymer concentration and $C_{b}$ is the salt concentration.

In this study, the function relationship represented by (6) is given by experimental data. Namely, a series of $\mu_{p}$ values corresponding to various polymer concentrations are given for different salinities, and $\mu_{p}$ is determined by linearly interpolating in the closed interval from 0 to the maximum given value of $C_{p}$ and $C_{s a}$.

Secondly, the effective water phase viscosity is calculated as follows:

$$
\mu_{e f f}=\mu_{w}+M \cdot\left(\mu_{0}-\mu_{w}\right)
$$

where $\mu_{\text {eff }}$ is the water phase viscosity with shear thinning effect. $M$ is a multiplier representing the shear thinning property of the water phase, which is given by

$$
M=\frac{\mu_{\mathrm{eff}}-\mu_{w}}{\mu_{0}-\mu_{w}}
$$

where $0<M<1$ which is equivalent to $\mu_{w}<\mu_{\text {eff }}<\mu_{0}$.

Similar approaches for the determination of the water phase viscosity have been used by other researchers in numerical studies or commercial simulators for polymer flooding $[1,20]$.

2.3. Modeling the Polymer In Situ Rheology. Most polymers used in EOR are pseudoplastic fluids which exhibits shear thinning behaviors [2, 4]. For pseudoplastic fluids, several common types of rheology models are given as below.

Power-law model is a simple and common used rheology model to describe the non-Newtonian rheology, for which (9) is the constitutive equation and (10) gives the effective viscosity [11]:

$$
\begin{gathered}
\tau=K \gamma^{n}, \\
\mu_{\mathrm{eff}}=K \gamma^{n-1},
\end{gathered}
$$

where $\mu_{\text {eff }}$ is the effective viscosity, $K$ is the consistency index, $\gamma$ is the shear rate, and $n$ is the power law index.

Another commonly used formula to describe the relationship between viscosity and the shear rate is Meter's equation [8], for which the constitutive equation is

$$
\gamma=\frac{\tau}{\mu_{\infty}+\left(\mu_{0}-\mu_{\infty}\right) /\left(1+\left(\dot{\gamma} / \dot{\gamma}_{1 / 2}\right)^{P_{\alpha}-1}\right)}
$$

and the effective viscosity is given by

$$
\mu_{\mathrm{eff}}=\mu_{\infty}+\frac{\mu_{0}-\mu_{\infty}}{1+\left(\dot{\gamma} / \dot{\gamma}_{1 / 2}\right)^{P_{\alpha}-1}}
$$

where $\mu_{\infty}$ is the viscosity when shear rate tends to infinity. $\dot{\gamma}_{1 / 2}$ is the shear rate at which the viscosity equals $\left(\mu_{o}+\mu_{\infty}\right) / 2$ and $P_{\alpha}$ is a constant.

Various empirical parameters such as $P_{\alpha}$ and $\dot{\gamma}_{1 / 2}$ are involved if we induce the models of (9)-(12) into the numerical calculation. However, these parameters are difficult to determine in some cases. Therefore, an alternate method which is similar to the treatment of commercial simulators [20] is used in this study, in which the $M-V$ relation is given directly to calculate the non-Newtonian viscosity, where $V$ stands for the flow velocity and $M$ is the shear thinning multiplier defined by (8). The $M-V$ relation indicates the shear thinning properties of non-Newtonian polymer solution.

\section{Results and Discussion}

In this part, a $2000 \times 2000 \times 10 \mathrm{~m}$ single-layer homogeneous reservoir is studied. Two wells are located in the reservoir, as shown in Figure 1. The production well produces at a constant liquid rate of $10 \mathrm{~m}^{3} /$ day at surface condition for 30 days. The injection well injects polymer solution at a constant liquid rate of $10 \mathrm{~m}^{3} /$ day at surface condition for 20 days and then shuts in for 10 days. Reservoirs parameters for calculation are listed in Table 1. Table 2 gives the relative permeability relation. Table 3 gives the relation between water phase unsheared viscosity (viscosity in the stationary situation) and polymer concentration for salinity equals 3000 . Table 3 gives the relationship between the viscosity multiplier $\mu_{p}$ and polymer concentration $C_{p}$, which is obtained from experiments on the polymers used in oilfield. The polymer solution is prepared using HPAM of 25 million molecular weight and treated produced water with a total salinity of $3000 \mathrm{mg} / \mathrm{L}$.

3.1. Pressure Transient Response for Various Polymer Concentrations without Consideration of the Shear Thinning Effect. In this part, the impact of polymer thickening effect on BHP (bottom-hole pressure) is studied. To eliminate the influent of polymer shear thinning, here we make assumption that the polymer solution behaves as Newtonian type fluid. Figure 2 gives the calculated BHP of the injection well for different injection polymer concentrations. Each of the pressure curves in Figure 2 includes two flow regimes: 0 to 20th day is the injection stage and 20th to 30th day the pressure fall-off stage. Figure 2 shows that for the first flow regime, the injection pressure becomes larger as the polymer concentration increases. This is due to the reduction of flow mobility near the injection well caused by the polymer concentration increase.

For the pressure fall-off regime, Figure 3 gives the loglog plot of the pressure change and pressure derivative for different injection polymer concentrations. The derivative curves in Figure 3 indicate that the increase of polymer concentration leads to a larger pressure change, which is 
TABLE 1: Reservoir parameters.

\begin{tabular}{lc}
\hline Parameters & Value \\
\hline Initial reservoir pressure, $\mathrm{MPa}$ & 20 \\
Layer thickness, m & 10 \\
Horizontal permeability, $\mu \mathrm{m}^{2}$ & 0.5 \\
Porosity & 0.2 \\
Compressibility of rock & 0.00015 \\
Initial saturation of oil & 0.3 \\
Initial saturation of water & 0.7 \\
Initial concentration of polymer, $\mathrm{kg} / \mathrm{m}^{3}$ & 0 \\
Wellbore radius, $\mathrm{m}$ & 0.1 \\
Liquid rate of production well, $\mathrm{m}^{3} / \mathrm{day}$ & 10 \\
Liquid rate of injection well, $\mathrm{m}^{3} / \mathrm{day}$ & 10 \\
Reference pressure for PVT, $\mathrm{MPa}$ & 20 \\
Viscosity of oil, Pa·s & 0.01 \\
Viscosity of water, Pa·s & 0.0006 \\
Volume factor for oil & 1.12 \\
Volume factor for water & 1.01 \\
Compressibility of oil, $1 / \mathrm{MPa}$ & 0.0006 \\
Compressibility of water, $1 / \mathrm{MPa}$ & 0.0006 \\
\hline
\end{tabular}

TABLE 2: Relation between the water phase saturation and relative permeability.

\begin{tabular}{ccc}
\hline$S_{w}$ & $k_{r w}$ & $k_{r o}$ \\
\hline 0.3 & 0 & 1 \\
0.4 & 0.02 & 0.72 \\
0.5 & 0.05 & 0.44 \\
0.6 & 0.09 & 0.17 \\
0.7 & 0.15 & 0.05 \\
0.8 & 0.24 & 0 \\
\hline
\end{tabular}

TABLE 3: Relation between polymer concentration and water phase viscosity.

\begin{tabular}{lc}
\hline Polymer concentration $\left(\mathrm{kg} / \mathrm{m}^{3}\right)$ & Viscosity multiplier \\
\hline 0 & 1 \\
0.5 & 4 \\
1 & 8 \\
1.5 & 13 \\
2 & 21 \\
\hline
\end{tabular}

$0.19673 \mathrm{MPa}, 0.47324 \mathrm{MPa}$, and $0.94508 \mathrm{MPa}$ for polymer concentration equals $0,0.5, \mathrm{~kg} / \mathrm{m}^{3}$, and $1.0 \mathrm{~kg} / \mathrm{m}^{3}$, respectively. This is caused by the polymer thickening effect. The growth of injection polymer concentration reduces the mobility of fluid near the injector, thus leading to a larger $\mathrm{BHP}$ rise while injecting at a constant liquid rate. Accordingly, after the well shuts in, the pressure drop of the injection well is also larger. Moreover, as the fluid mobility reduces, the flow-continued time of the wellbore storage regime and the transition stage becomes much longer.

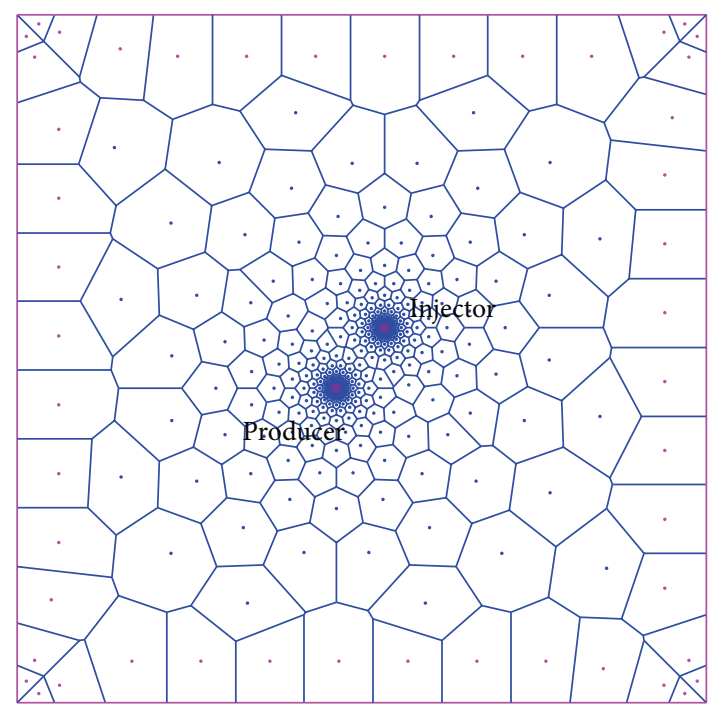

FIGURE 1: Computational domain and gridding.

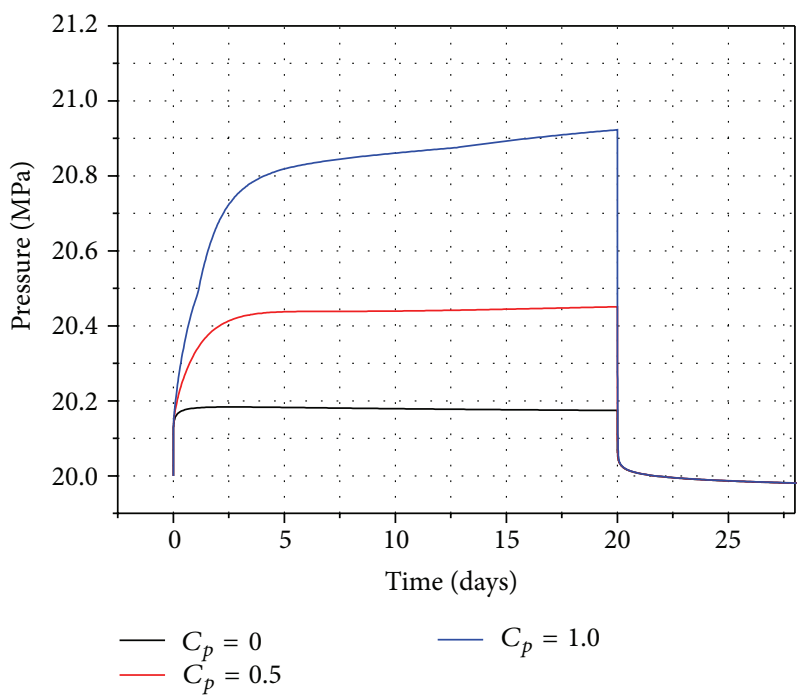

FIGURE 2: BHP of the injection well for different injection polymer concentrations.

3.2. Impact of Shear Thinning Effect on Pressure Transient Response. To analyse the pressure transient response of polymer flooding wells, the shear thinning effect needs to be considered. As is discussed in the previous part, the $M-V$ relation should be given to determine the effective viscosity of water phase with (8). Here, we make assumption that the shear rate of flow in the reservoir is proportional to the water phase velocity, and another simplification is made that the $M-V$ relation is not influenced by the polymer concentration. Three $M-V$ curves used for the calculation in this example are given in Figure 4. It is obvious that the black curve in Figure 4 has the highest rate of descent which indicates a stronger influence of water phase velocity on viscosity. Accordingly, the red curve represents a medium one and the blue curve a small one. The three curves are manually generated according to the following rules: (1) the multiplier $M$ changes from 1 to 0 


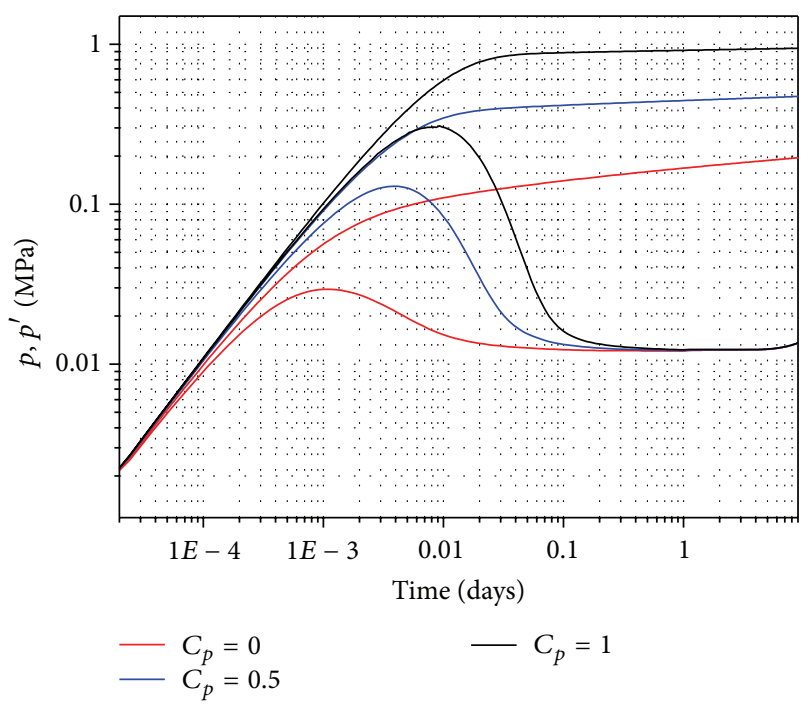

FIGURE 3: Log-log plot of the well bottom pressure and pressure derivative of the pressure fall-off period for various injection polymer concentrations.

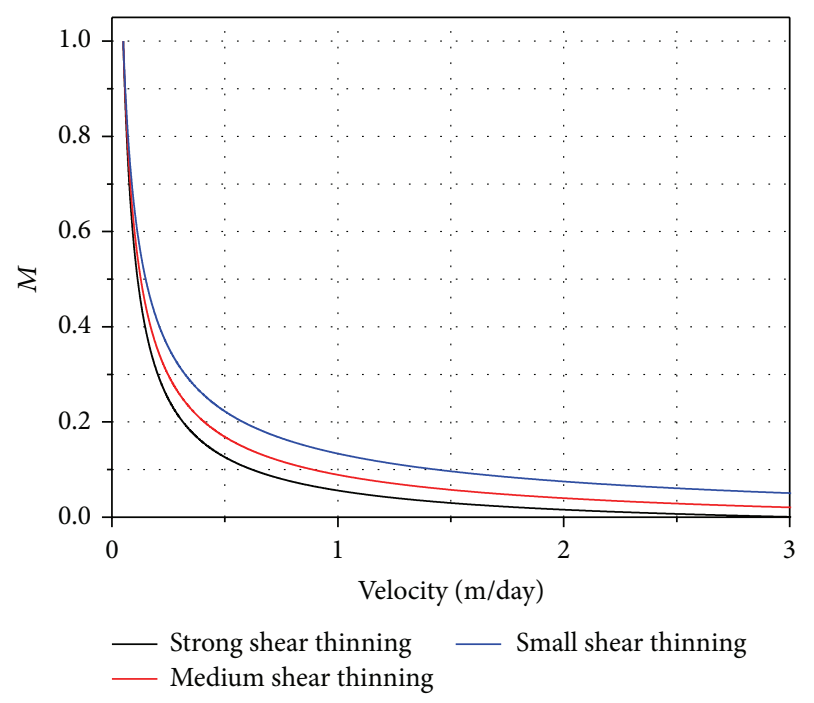

FIgURE 4: $M-V$ curves.

with the velocity increases. (2) All curves go through the same point $(0,1)$ which means when the velocity is 0 , the effective viscosity equals the unsheared viscosity $\mu_{0}$. (3) As the velocity increases the value of $M$ tends to 0 , which means the effective viscosity goes close to the pure water viscosity.

Figure 5 shows the comparison of BHP calculated under different shear thinning curves and a Newtonian situation, with the same polymer concentration of $1.5 \mathrm{~kg} / \mathrm{m}^{3}$. A significant decrease of BHP is showed by the comparison between the results calculated with and without shear thinning, and the BHP calculated under a strong shear thinning is lower than that under a slight one. A qualitative explanation is that shear thinning reduces the effective viscosity of injected polymer solution and makes the injection pressure much lower.

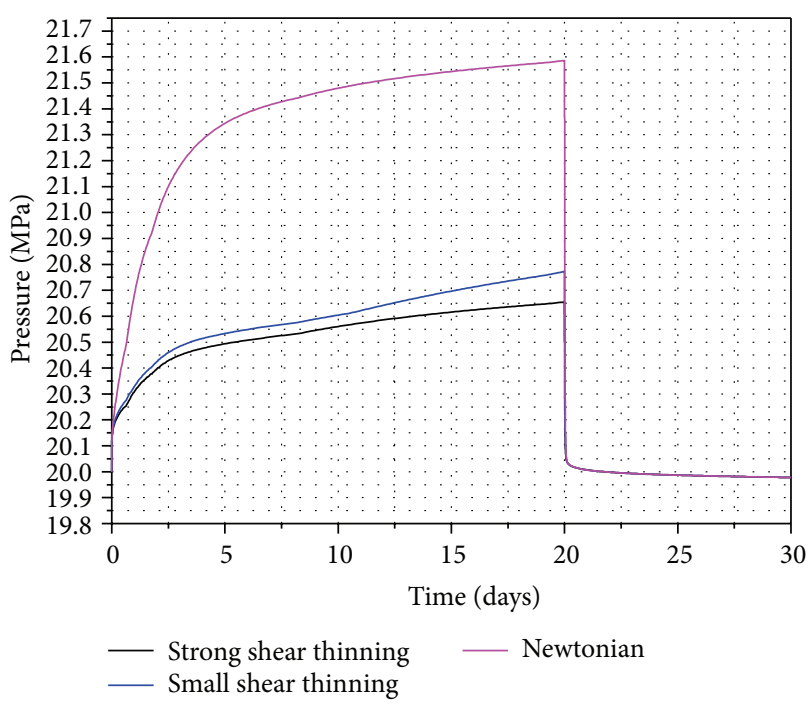

Figure 5: BHP of the injection well for different $M-V$ curves with the same injection polymer concentration of $1.5 \mathrm{~kg} / \mathrm{m}^{3}$.

For the fall-off regime, Figure 6 gives the comparison of pressure change and derivative under different shear thinning behaviors and the Newtonian situation. It is showed in the figure that when shear thinning is considered, the pressure change decreases significantly; the stronger the shear thinning is, the smaller the pressure change becomes. This can be explained by considering the impact of shear thinning on the water phase mobility, which is similar to the previous explanation for Figure 3. But we find that Figure 6 differs from Figure 3 in that the flow-continued time of the wellbore storage regime and the transition stage is not significantly influenced by shear thinning. The reason is that the influent of shear thinning is velocity dependent. In the fall-off regime, the sheared viscosity is small at the beginning but increases quickly as the flow slows down until the viscosity value is close to unsheared viscosity. This process will not last long, which makes the flow-continued time under shear thinning still shorter than but not significantly different from that of the Newtonian situation.

An impressive phenomenon we find from Figure 6 is that the pressure derivative curves are nonsmooth in early stage when we consider the shear thinning effect. The nonsmoothness of the derivative curves, as we consider, is caused by the rapid change of near-wellbore mobility due to the shear thinning behavior. A qualitative explanation is that when shear thinning exists, the sudden decrease of flow speed after well shuts in causes a dramatic change of water phase viscosity, and this gives a disturbance to BHP, changing the original trend of pressure drop. The disturbance of BHP is more detectable on the derivative which becomes an obvious and characteristic nonsmoothness at early stage. The nonsmoothness, as caused by the shear thinning behavior, is defined as the shear-thinning-induced nonsmoothness in the paper.

We also find that the oscillation amplitude of the nonsmoothness is affected by relevant factors such as the shear thinning curve and the polymer concentration. Figure 6 


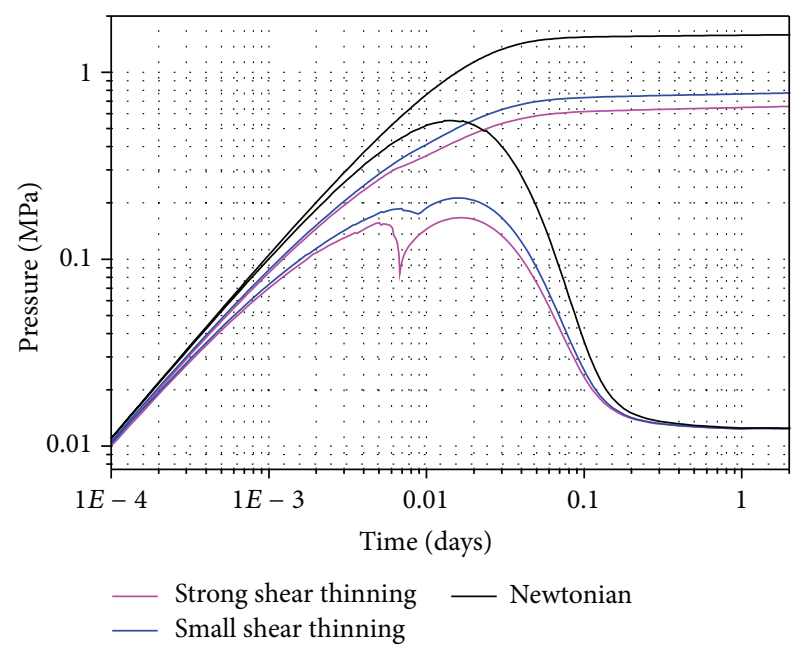

FIgURE 6: Log-log plot of the well bottom pressure and pressure derivative of the pressure fall-off period for different $M-V$ curves with the same injection polymer concentration of $0.7 \mathrm{~kg} / \mathrm{m}^{3}$.

shows that the stronger the shear thinning is, the more obvious the nonsmoothness becomes. Figure 7 shows the impact of polymer concentration on pressure transient response with shear thinning effect and the results indicate that the nonsmoothness also becomes more strenuous under a higher polymer concentration.

Other factors we do not consider here may also have influence on the shear-thinning-induced nonsmoothness of derivative curves, which has been observed in field cases, for example, the alternating injection of polymers with different molecular weights. In addition, we do not study the effect of adsorption, inaccessible porosity, and permeability reduction on the transient pressure response in this paper, although they are incorporated in the mathematical model.

It should be noted that consideration of the shear thinning makes the water phase viscosity dependent on velocity, which may greatly increase the difficulty of convergency of the iterative solving and makes the simulation more time consuming.

3.3. Transient Pressure Response for Production Well. We also study the pressure transient response of production well in a build-up test in the same example. The injection well injects at a constant liquid rate of $10 \mathrm{~m}^{3} /$ day at surface condition for 30 days. The production well produces solution at the same liquid rate of $10 \mathrm{~m}^{3} /$ day at surface condition for 20 days and then shuts in for 10 days. Reservoirs parameters are the same as those in the previous fall-off case. In order to facilitate the research, we assume that there was a uniform distribution of polymer at the beginning of the simulation. The original polymer concentration of the reservoir is $0.8 \mathrm{~kg} / \mathrm{m}^{3}$, and the injected polymer concentration is also $0.8 \mathrm{~kg} / \mathrm{m}^{3}$.

Figure 8 gives the comparison of transient pressure responses calculated under the Newtonian and the nonNewtonian situation, respectively. The result of the buildup test indicates similar conclusion to that of a fall-off

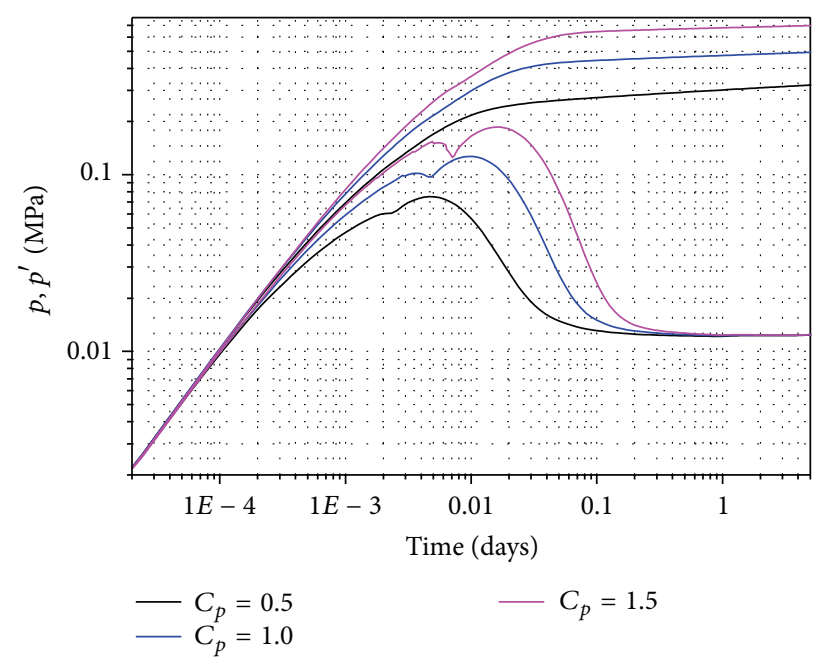

FIGURE 7: Log-log plot of the well bottom pressure and pressure derivative of the pressure fall-off period for various polymer concentrations under the medium shear thinning.

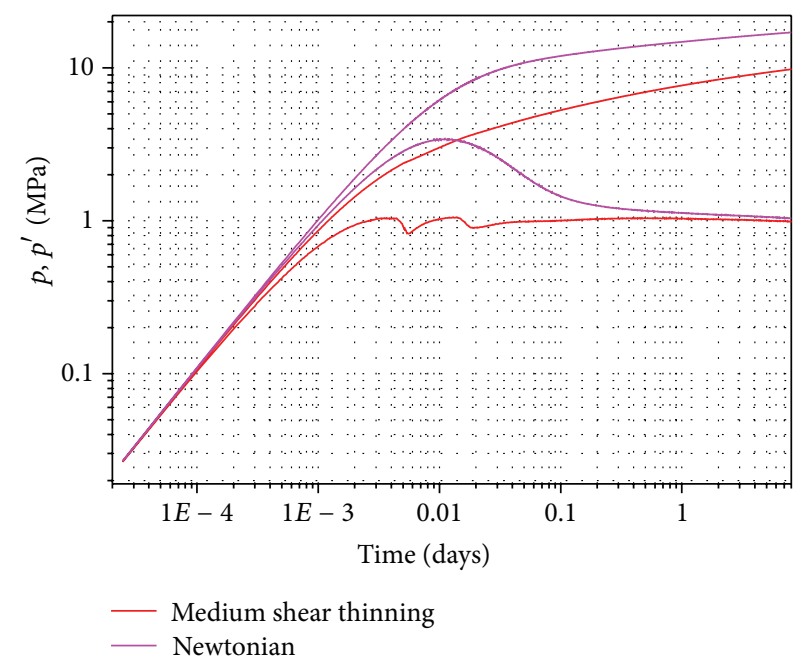

FIGURE 8: Log-log plot of the well bottom pressure and pressure derivative of the production well for different $M-V$ curves.

test: shear thinning reduces the pressure change and draws down the peak value of derivative and induces early stage nonsmoothness on the derivative curve. As there exists an original distribution of polymer, the curves in Figure 8 are different from those in Figure 6 in late stage.

\section{Case Study}

We study a five-well group from a polymer flooding reservoir in Daqing oil field with a production well in the center, and other four injection wells around it. The average layer thickness is $5.5 \mathrm{~m}$. The positions of the wells are shown in Figure 9. Water flooding production lasts for years in the reservoir before the polymer flooding stage, and polymer injection started in October 2009. 


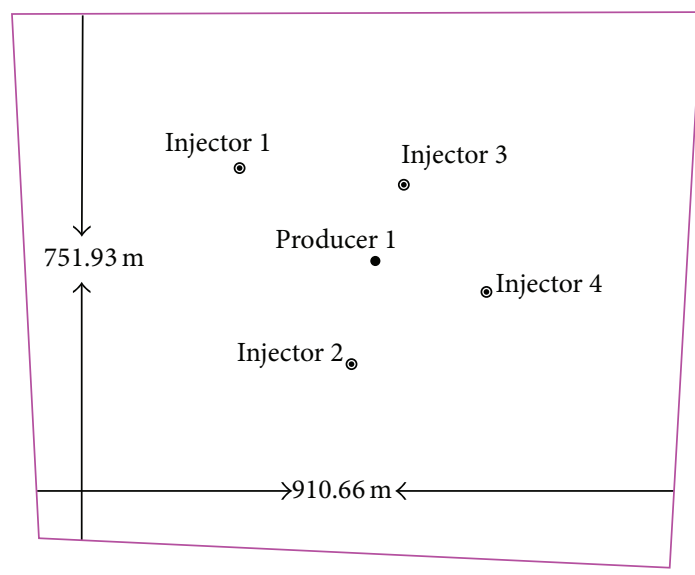

FIgURE 9: Computational domain for the real example.

Two pressure build-up tests were performed for the oil producer in 2010.04 and 2012.04, respectively, for which the details are given in Table 4. Log-log plots of the pressure change and pressure derivative curves for the two well tests are demonstrated in Figures 11 and 12.

We can see from Figures 10 and 11 that pressure transient response of the two build-up tests is different in that the derivative curves of Testl is smooth while the derivative curve of Test 2 shows early stage nonsmoothness. We explain this with the shear-thinning-induced nonsmoothness. Here are some evidences. By referring to the record of production, we know that polymer injection began in 2009.10. Polymer concentration detected at the production well stays very low at first until a marked increase in 2010.07, and polymer concentration by the time of 2010.04 is only $0.009 \mathrm{~kg} / \mathrm{m}^{3}$ which means the injected polymer solution had not reached the production well in 2010.4. Therefore, pressure derivative curve of Testl is not affected by the polymer shear thinning and shows typical characteristic of Newtonian flow situation. While for Test2, by the time of which the measured polymer concentration at the production well is $0.85 \mathrm{~kg} / \mathrm{m}^{3}$. So according to the discussion of the example given above, it is possible that shear thinning of polymer solution affects the derivative curve of Test2, making it nonsmooth in the early stage.

We design numerical calculation and perform well test interpretation for the measured transient pressure data. The calculation domain and gridding are shown in Figure 12, and other parameters for calculation are given in Table 5. The matched results of the measured pressure data and numerical calculation data are shown in Figures 13 and 14. The interpreted shear thinning curve is shown in Figure 15, and other interpreted reservoir parameters are given in Table 6.

The result of numerical simulation indicates that by the beginning of well test 1 , polymer concentration in the production well is 0 , which means that injected polymer had not reached the production well. Figure 13 shows that the measured pressure change and the derivative curve of the pressure are well matched by the numerical result. We note that the calculated derivative curves of Testl are smooth in early stage. For Test2, numerical result shows
TABLE 4: Details of the well tests.

\begin{tabular}{|c|c|c|}
\hline Name & Testl & Test2 \\
\hline Date of test & $2010 / 04 / 19$ & $2012 / 04 / 15$ \\
\hline Well & Producer 1 & Producer 1 \\
\hline Well test method & Pressure build-up & Pressure build-up \\
\hline $\begin{array}{l}\text { Time of stable } \\
\text { production, day }\end{array}$ & 150 & 150 \\
\hline $\begin{array}{l}\text { Liquid rate of stable } \\
\text { production, } \mathrm{m}^{3} / \text { day }\end{array}$ & 74 & 30 \\
\hline $\begin{array}{l}\text { Time of well shutdown, } \\
\text { day }\end{array}$ & 3.01 & 3.09 \\
\hline $\begin{array}{l}\text { Measured concentration } \\
\text { of polymer in the } \\
\text { production well, } \mathrm{kg} / \mathrm{m}^{3}\end{array}$ & 0.009 & 0.85 \\
\hline
\end{tabular}

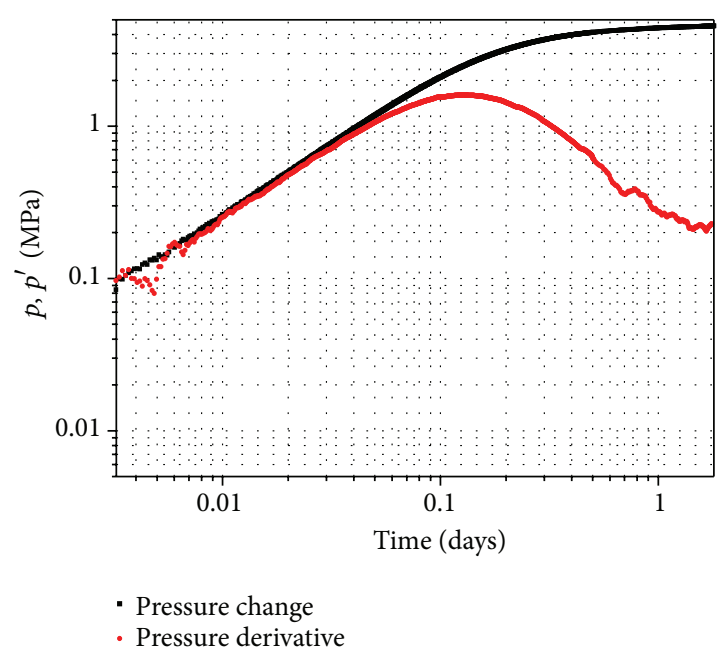

Figure 10: Measured pressure change and pressured derivative curves for Testl.

polymer concentration of the producer is $0.74 \mathrm{~kg} / \mathrm{m}^{3}$, while the measured data is $0.85 \mathrm{~kg} / \mathrm{m}^{3}$. The results indicate that polymer solutions injected into the reservoir had already reached the oil producer by the time of Test 2 . Figure 14 shows that not only the whole shape of the curves is well matched but also the nonsmoothness of the derivative is fitted through the interpretation. During the fitting for Test2 we find that the oscillation amplitude and position of the nonsmoothness are quite sensitive to the shear thinning curves, and small change of the shear thinning curve may lead to obvious change of the nonsmoothness. This indicates that in well test analysis the nonsmoothness of the derivative curve is a significative feature which can be interpreted to determine the shear thinning curve.

The finding shows significant importance for practical well test analysis for the following reasons: first, the nonsmoothness of pressure derivative curves is a common phenomenon of well test for polymer flooding in oilfields, and it is mostly considered to be caused by the heterogeneity of polymer slug, or measurement error. The finding of this study provides a new explanation for the frequently occurring of 
TABLE 5: Parameters for calculation.

\begin{tabular}{lc}
\hline Parameters & Value \\
\hline Layer thickness, $\mathrm{m}$ & 5.5 \\
Horizontal permeability, $\mu \mathrm{m}^{2}$ & $\begin{array}{c}\text { Permeability } \\
\text { distribution }\end{array}$ \\
Porosity (initial) & 0.2 \\
Compressibility of rock, 1/MPa & 0.00015 \\
Initial saturation of oil & 0.35 \\
Initial saturation of water & 0.65 \\
Wellbore radius, m & 0.1 \\
Reference pressure for PVT Calculation, MPa & 2 \\
Viscosity of oil, Pa·s & 0.0067 \\
Viscosity of water, Pa·s & 0.0006 \\
Volume factor for oil & 1.12 \\
Volume factor for water & 1 \\
Compressibility of oil, 1/MPa & 0.00084 \\
Compressibility of water, 1/MPa & 0.000449 \\
\hline
\end{tabular}

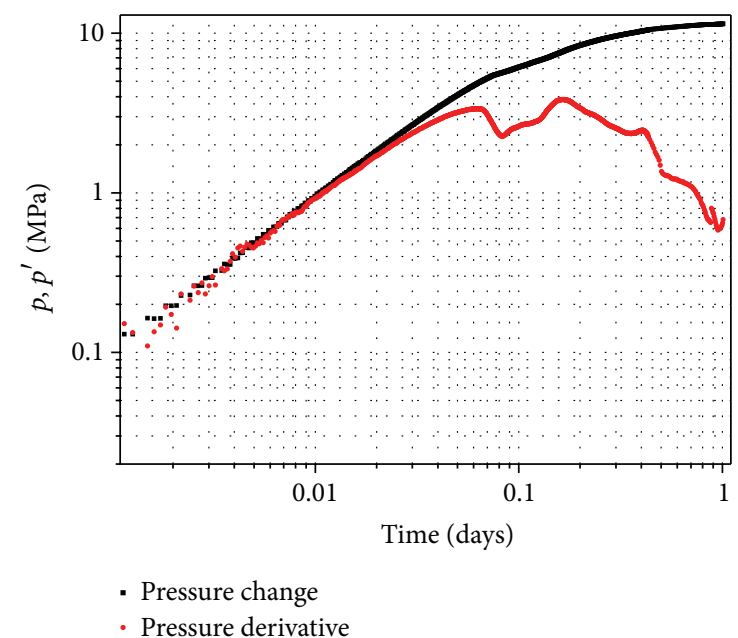

FIgURE 11: Measured pressure change and pressured derivative curves for Test 2 .

derivative curve nonsmoothness. Second, while most shear thinning data used in oil field are obtained from simplified experiments and do not represent the real in situ rheology, this finding provides an approach to estimate the polymer in situ rheology from transient pressure data.

Other interpreted parameters in Table 6 show that the near-wellbore permeability decreased and skin increased from Test 1 to Test2. The interpreted data in Table 6 reflect the damage of the formation and wellbore by polymer solution.

\section{Conclusion}

In this paper, a polymer flooding model is presented and a fully implicit numerical simulator was developed based on the PEBI grid. Using the numerical simulator, parametric studies are conducted to study the pressure transient analysis for polymer flooding. Finally, a field case is studied and

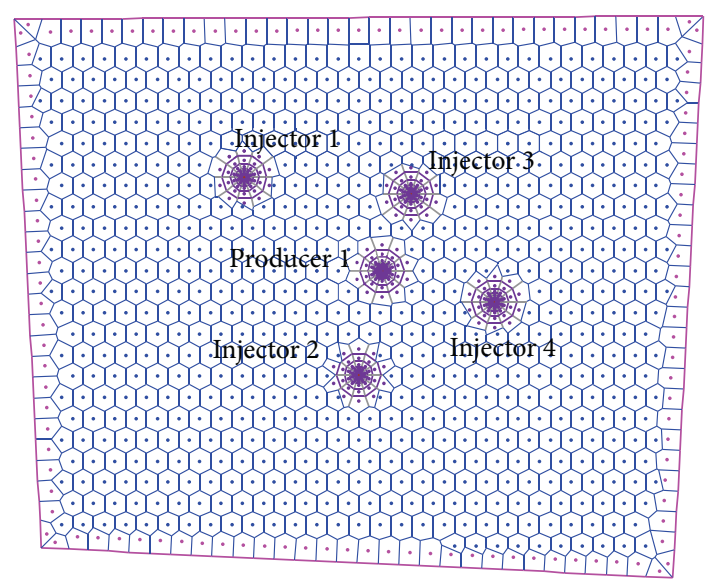

FIGURE 12: Gridding for the real example.

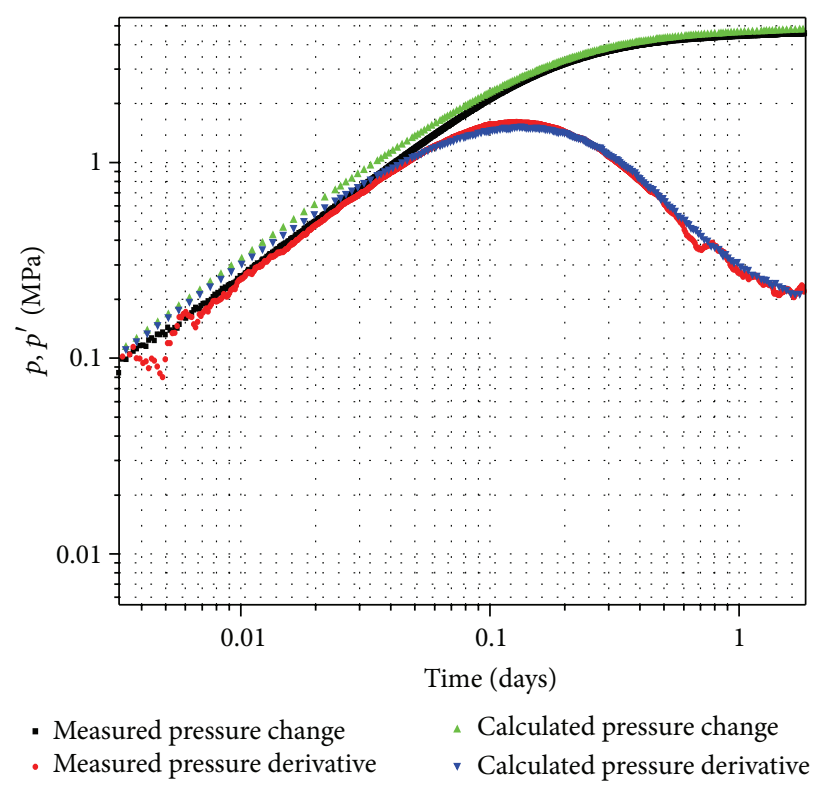

FIGURE 13: Pressure change and pressure derivative history match for Test1.

numerical well test analysis is performed to interpret the measured data.

By performing parametric studies, we find that the polymer concentration and polymer shear thinning effect greatly affect the BHP. The pressure transient response under different polymer concentration without consideration of the shear thinning effect show that the increasing polymer concentration makes a larger pressure change, and a longer flow-continued time of the wellbore storage stage and the transition stage. By considering the shear thinning effect, we find that the polymer shear thinning leads to a smaller pressure change, and the flow-continued time of the early stage is not significantly affected by shear thinning effect. Shear thinning also makes the pressure derivative curve nonsmooth during the wellbore storage and the transition stages, which is caused by the rapid change of the fluid mobility. The oscillation amplitude of the nonsmoothness is 
TABLE 6: Interpreted parameters.

\begin{tabular}{lcccc}
\hline Date & Near-wellbore permeability, $\mu \mathrm{m}^{2}$ & Wellbore storage, $\mathrm{m}^{3} / \mathrm{MPa}$ & Skin & Porosity \\
\hline 2010.4 & 0.35 & 2.65 & 4.8 & 0.2 \\
2012.4 & 0.25 & 0.6 & 9 & 0.2 \\
\hline
\end{tabular}

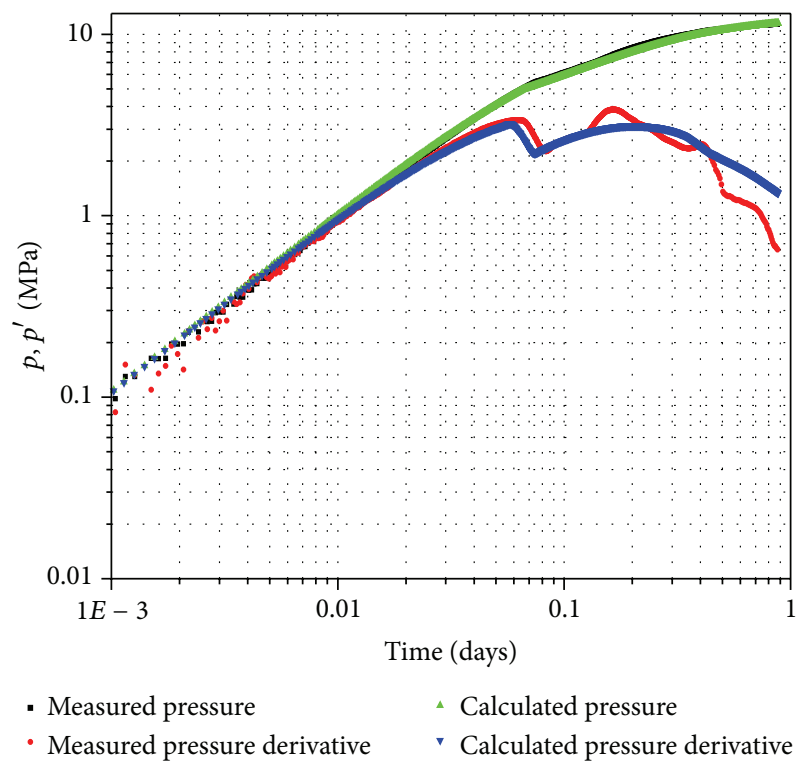

FIgURE 14: Pressure change and pressure derivative history match for Test2.

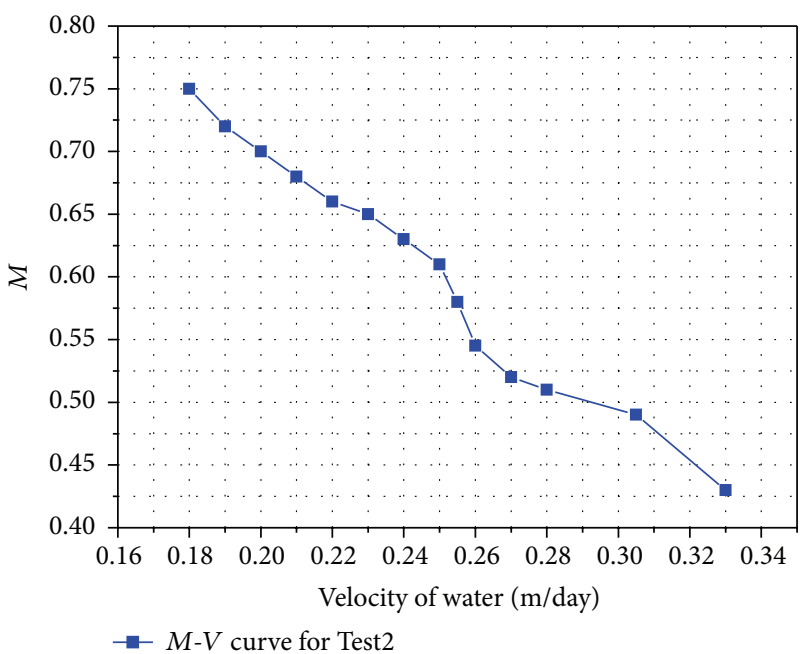

FIGURE 15: Interpreted $M-V$ shear thinning curve.

influenced by the $M-V$ shear thinning curve and the polymer concentration.

A field case is studied in which two pressure buildup tests were performed on an oil producer in different stages of polymer flooding process. Significant differences are detected between the measured pressure curves of the two well tests, among which the pressure derivative curve of the later one showed noticeable feature of polymer shear thinning while the derivative curve of the earlier well test does not. The measured pressure data validate our parametric study. Numerical calculation and well test interpretation are also performed for the field example. It is found that the shear thinning calculation has a significant impact on fitting of the transient pressure curves, and the nonsmoothness of pressure derivative is possibe to be interpreted to determine the polymer in situ non-Newtonian properties.

\section{Nomenclature}

B: $\quad$ Formation volume, dimensionless

C: $\quad$ Mass concentration, $\mathrm{kg} / \mathrm{m}^{3}$

$C_{\text {ads }}: \quad$ Adsorbed concentration, $\mathrm{kg} / \mathrm{kg}$

$C_{\text {wellbore }}$ : Wellbore storage, $\mathrm{m}^{3} / \mathrm{MPa}$

D: $\quad$ Depth, m

$h$ : $\quad$ Layer thickness, $m$

$K: \quad$ Absolute permeability, $\mathrm{m}^{2}$

$K_{r}: \quad$ Relative permeability, dimensionless

$M: \quad$ Shear thinning multiplier, dimensionless

n: $\quad$ Time step, dimensionless

p: $\quad$ Pressure, $\mathrm{Pa}$

$q$ : $\quad$ Flow rate, $\mathrm{m}^{3} / \mathrm{s}$

$R_{k}$ : Permeability reduction factor, dimensionless

$r_{w}: \quad$ Wellbore radius, $\mathrm{m}$

$r_{\text {eff: }} \quad$ Effective radius, $m$

$S_{l}$ : $\quad$ Saturation of phase, $l$ subscript $l=o, w$, dimensionless

S: $\quad$ Skin factor, dimensionless

$t: \quad$ Mass concentration, $s$

$V: \quad$ Velocity, $\mathrm{kg} / \mathrm{m}^{3}$

$\gamma: \quad$ Shear rate, $\mathrm{s}^{-1}$

$\gamma_{l}: \quad$ Gravity factor, $\mathrm{N} / \mathrm{m}^{3}$

$\mu$ : Viscosity, Pa.s.

\section{Subscripts}

$o$ : Oil phase

$w$ : Water phase

$p$ : Polymer

sa: Salt

0 : Zero shear rate.

\section{Conflict of Interests}

The authors declare that there is no conflict of interests regarding the publication of this paper. 


\section{Acknowledgments}

This work was sponsored by Major State Basic Research Development Program of China (973 Program) (no. 2011CB707305), National Key Science and Technology Project (2011ZX05009-006), and CAS Strategic Priority Research Program (XDB10030402).

\section{References}

[1] A. M. AlSofi and M. J. Blunt, "Streamline-based simulation of non-Newtonian polymer flooding," SPE Journal, vol. 5, no. 4, pp. 895-905, 2010.

[2] L. W. Lake, Enhanced Oil Recovery, Prentice Hall, Englewood Cliffs, NJ, USA, 1989.

[3] S. Vongvuthipornchai and R. Raghavan, "Pressure falloff behavior in vertically fractured wells: non-newtonian power-law fluids. spe formation evaluation," SPE Formation Evaluation, vol. 2, no. 4, pp. 573-589, 1987.

[4] A. M. AlSofi, T. C. LaForce, and M. J. Blunt, "Sweep impairment due to polymers shear thinning," in Proceedings of the 16th Middle East Oil and Gas Show and Conference (MEOS '09), pp. 834-845, Bahrain, Bahrain, March 2009.

[5] X. Lopez and M. J. Blunt, "Predicting the impact of nonnewtonian rheology on relative permeability using pore-scale modeling," in Proceedings of the SPE Annual Technical Conference and Exhibition, Paper SPE 89981, pp. 793-800, Houston, Tex, USA, September 2004.

[6] B. Wang, L. W. Lake, and G. A. Pope, "Development and application of a streamline micellar/polymer simulator," in Proceedings of the SPE Annual Technical Conference and Exhibition, Paper SPE10290, San Antonio, Tex, USA, October 1981.

[7] A. Laoroongroj, M. Zechner, T. Clemens, and A. Gringarten, "Determination of the in-situ polymer viscosity from fall off tests," in Proceedings of the SPE Europec/EAGE Annual Conference, Paper SPE 154832, Copenhagen, Denmark, June 2012.

[8] D. M. Meter and B. R. Bird, "Tube flow of non-Newtonian polymer solutions, parts 1 and 2-laminar flow and rheological models," American Institute of Chemical Engineer, vol. 878-881, pp. 1143-1150, 1964.

[9] J. G. Savins, "Non-newtonian flow through porous media," Industrial \& Engineering Chemistry, vol. 61, no. 10, pp. 18-47, 1969.

[10] W. B. Gogarty, G. L. Levy, and V. G. Fox, "Viscoelastic effects in polymer flow through porous media," in Proceedings of the Fall Meeting of the Society of Petroleum Engineers of AIME, Paper SPE 4025, San Antonio, Tex, USA, October 1972.

[11] X. Y. Kong, Transport in Porous Media, Printing House of University of Science and Technology of China, Hefei, China, 2010 (Chinese).

[12] C. Huh and T. Snow, "Well testing with a non-Newtonian fluid in the reservoir," in Presented at the SPE Annual Technical Conference and Exhibition, Las Vegas, Nev, USA, September 1985, Paper SPE14453.

[13] I. Katime-Meindl and D. Tiab, "Analysis of pressure transient test of non-newtonian fluids in infinite reservoir and in the presence of a single linear boundary by the direct synthesis technique," in Proceedings of the SPE Annual Technical Conference and Exhibition, Paper SPE 71587, pp. 2213-2222, New Orleans, Lo, USA, October 2001.
[14] C. U. Ikoku and H. J. Ramey Jr., "Transient flow of nonNewtonian power-law fluids in porous medium," SPE Journal, vol. 19, no. 3, pp. 164-174, 1979, SPE 7139-PA.

[15] A. S. Odeh and H. T. Yang, "Flow of non-newtonian power-law fluids through in porous medium," SPE Journal, vol. 19, no. 3, pp. 155-163, 1979, SPE 7150-PA.

[16] S. Vongvuthipornchai and R. Raghavan, "Well test analysis of data dominated by storage and skin: non-Newtonian powerlaw fluids," SPE Formation Evaluation, vol. 2, no. 4, pp. 618-628, 1987, SPE14454-PA.

[17] F.-H. Escobar, J.-A. Martinez, and M. Montealegre-Madero, "Pressure and pressure derivative analysis for a well in a radial composite reservoir with a non-newtonian /newtonian interface," Ciencia, Tecnología y Futuro, vol. 4, no. 1, pp. 33-42, 2010.

[18] J. A. Martinez, F. H. Escobar, and M. Montealegre, "Vertical well pressure and pressure derivative analysis for bingham fluids in a homogeneous reservoirs," Dyna, vol. 78, no. 166, pp. 21-28, 2011.

[19] H. Y. Yu, H. Guo, Y. W. He et al., "Numerical well testing interpretation model and applications in crossflow double-layer reservoirs by polymer flooding," The Scientific World Journal, vol. 2014, Article ID 890874, 11 pages, 2014.

[20] H. Mahani, T. G. Sorop, P. J. van den Hoek, A. D. Brooks, and M. Zwaan, "Injection fall-off analysis of polymer flooding EOR," in Proceedings of the SPE Reservoir Characterisation and Simulation Conference and Exhibition, Paper SPE 145125, Abu Dhabi, UAE, October 2011. 

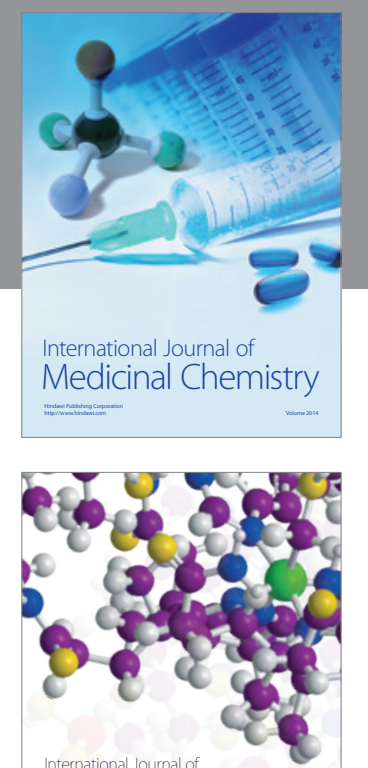

\section{Carbohydrate} Chemistry

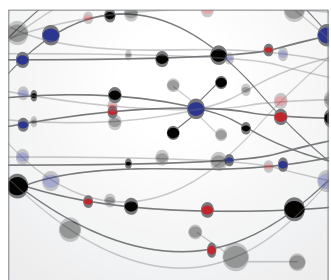

The Scientific World Journal
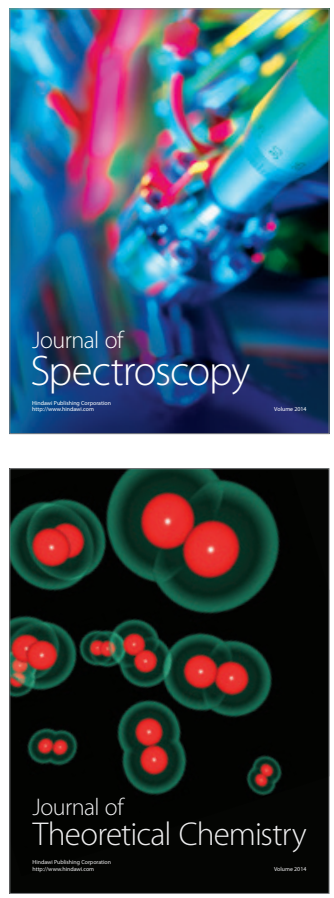
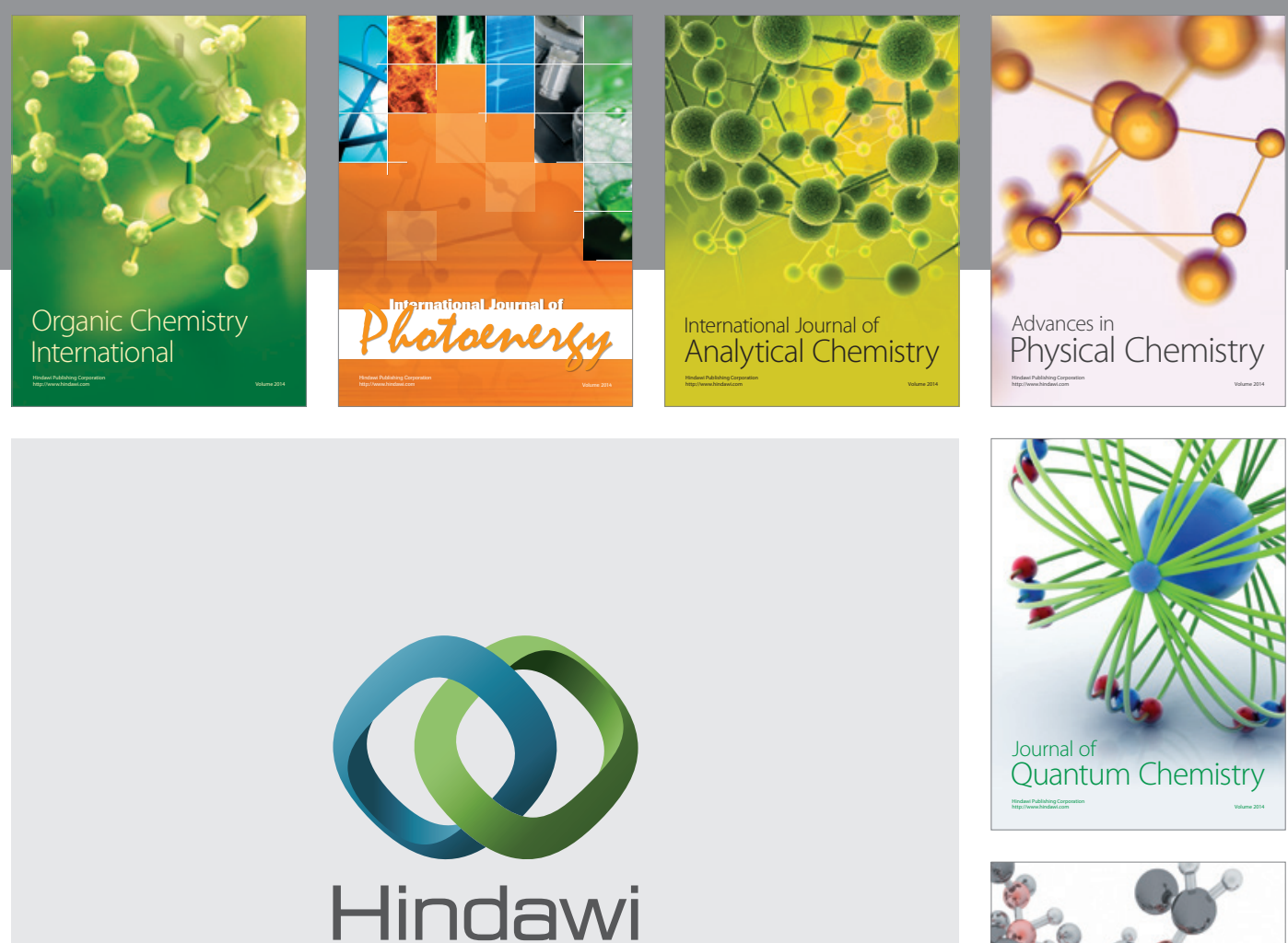

Submit your manuscripts at

http://www.hindawi.com

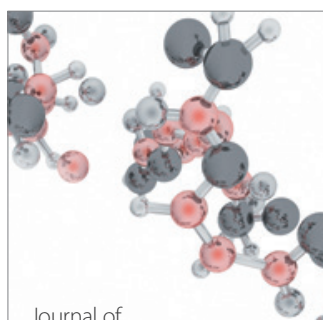

Analytical Methods

in Chemistry

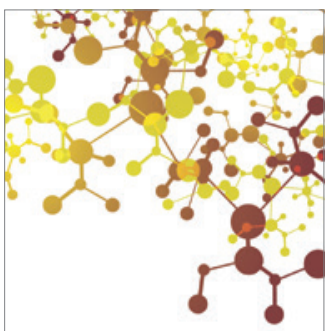

Journal of

Applied Chemistry

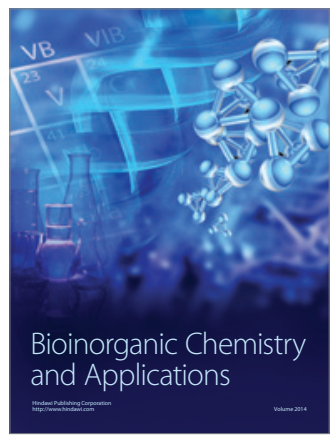

Inorganic Chemistry
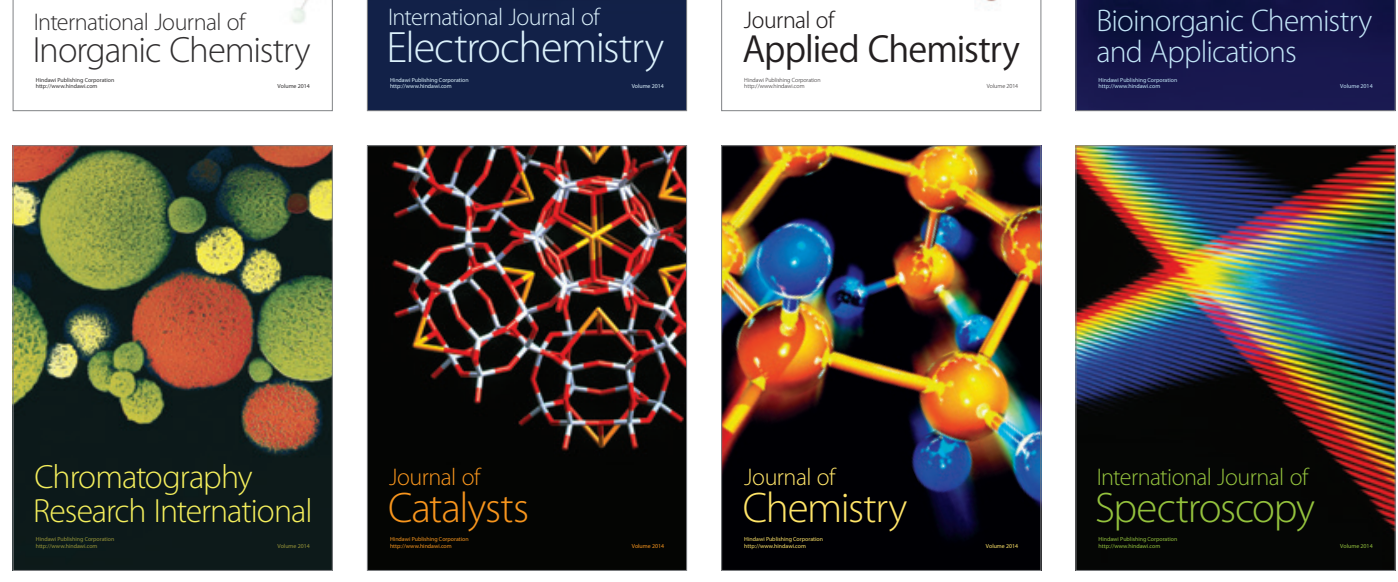